\title{
Microscopy of Plasma-Materials Interactions in Tungsten for Fusion Power
}

\author{
Chad M. Parish ${ }^{1}$, Russell P. Doerner ${ }^{2}$, Matthew J. Baldwin ${ }^{2}$, David Donovan ${ }^{3}$, Kevin G. Field ${ }^{1}$, and \\ Yutai Katoh ${ }^{1}$ \\ 1. Materials Science and Technology, Oak Ridge National Laboratory, Oak Ridge, TN USA \\ 2. Center for Energy Research, University of California San Diego, La Jolla, CA, USA \\ 3. Nuclear Engineering, University of Tennessee, Knoxville, TN, USA
}

Nuclear fusion reactors will require a first wall that can withstand extreme fluxes and fluences of impacting plasma ions (He, H), high temperatures, and a high-power flux of $14 \mathrm{MeV}$ neutrons. Tungsten is the material chosen for the first large-scale tokamak, ITER, but tungsten is insufficient for a full-scale fusion power plant. Materials engineering for this environment is still in its infancy; prior to materials design efforts, it is necessary to obtain the scientific underpinning of how plasma ions (particularly He) interact with the material and its defects. In this work, we use advanced electron microscopy to examine He-plasma-exposed polycrystalline tungsten as a means to explore the fundamental questions of plasmamaterials interactions.

Tungsten nanofuzz [1] grown at UC-San Diego PISCES-A $\left(900^{\circ} \mathrm{C}, 50 \mathrm{eV}, 4 \times 10^{26} \mathrm{He} / \mathrm{m}^{2}\right)$ was prepared by FIB milling using the ORNL FEI Versa DualBeam ${ }^{\mathrm{TM}}$ tool, and imaged using the ORNL FEI Talos S/TEM. FIB milling (Figure 1a) has the advantage that the fuzz / substrate interface is easily imaged in S/TEM (Figure 1b), and the sample is seen to contain a high density of inhomogeneous bubbles or cavities deep $(\sim 200 \mathrm{~nm})$ below the fuzz/substrate interface. This is surprising because He is introduced from the surface, and its transport mechanism to below $\sim 2000 \mathrm{~nm}$ tall fuzz is unknown. FIB has disadvantages, however: as shown in Figure 2, FIB artifacts (Pt, C, Ga) coat the tendrils and make it difficult to image their detailed internal structure.

By brushing a methanol-soaked carbon-filmed TEM grid across the fuzzy surface, individual tendrils can be extracted for S/TEM analysis (Figure 3, ORNL JEOL 2100F S/TEM). This allows more detailed examination of internal defects, such as grain boundaries and bubbles. Analysis of He pressure by EELS [2] has been shown on other materials, and is underway in this work. Individual grains in the tendrils can be analyzed by CBED in STEM mode, as has been applied to other nuclear materials problems [3], and characterization of the grain boundaries is presently underway (Figure 3).

For lower He fluxes and fluences, bubble structures in the substrate form prior to nanofuzz nucleation. An ALMT ITER-grade tungsten blank was exposed $\left(1000^{\circ} \mathrm{C}, 75 \mathrm{eV}, 3 \times 10^{24} \mathrm{He} / \mathrm{m}^{2}\right.$ [4]) and prepared for S/TEM (ORNL Talos) by FIB (ORNL Versa). TEM imaging shows defects, such as grain boundaries and dislocation loops, decorated with bubbles (Figure 4). This indicates that the starting structure will have a significant impact on the early stage helium disposition, and is under further study. [5]

[1] M.J. Baldwin, R.P. Doerner, Nucl. Fusion, V48 (2008), Art\# 035001.

[2] D. Taverna, Phys. Rev. Lett., V100 (2008), Art\# 035301.

[3] K. G. Field et al., J. Nucl. Mater., V435 (2013) P.172-180.

[4] D. Donovan et al., Phys. Script., V2016 (2016) P. T167.

[5] Work at ORNL supported by an Early Career Award, US Department of Energy, Office of Science, Fusion Energy Sciences. This research was performed, in part, using instrumentation (FEI Talos F200X S/TEM) provided by the Department of Energy, Office of Nuclear Energy, Fuel Cycle R\&D Program 
and the Nuclear Science User Facilities. Work at UC San Diego supported by DE-FG02-07ER54912. DD supported by DE-AC04-94AL85000.
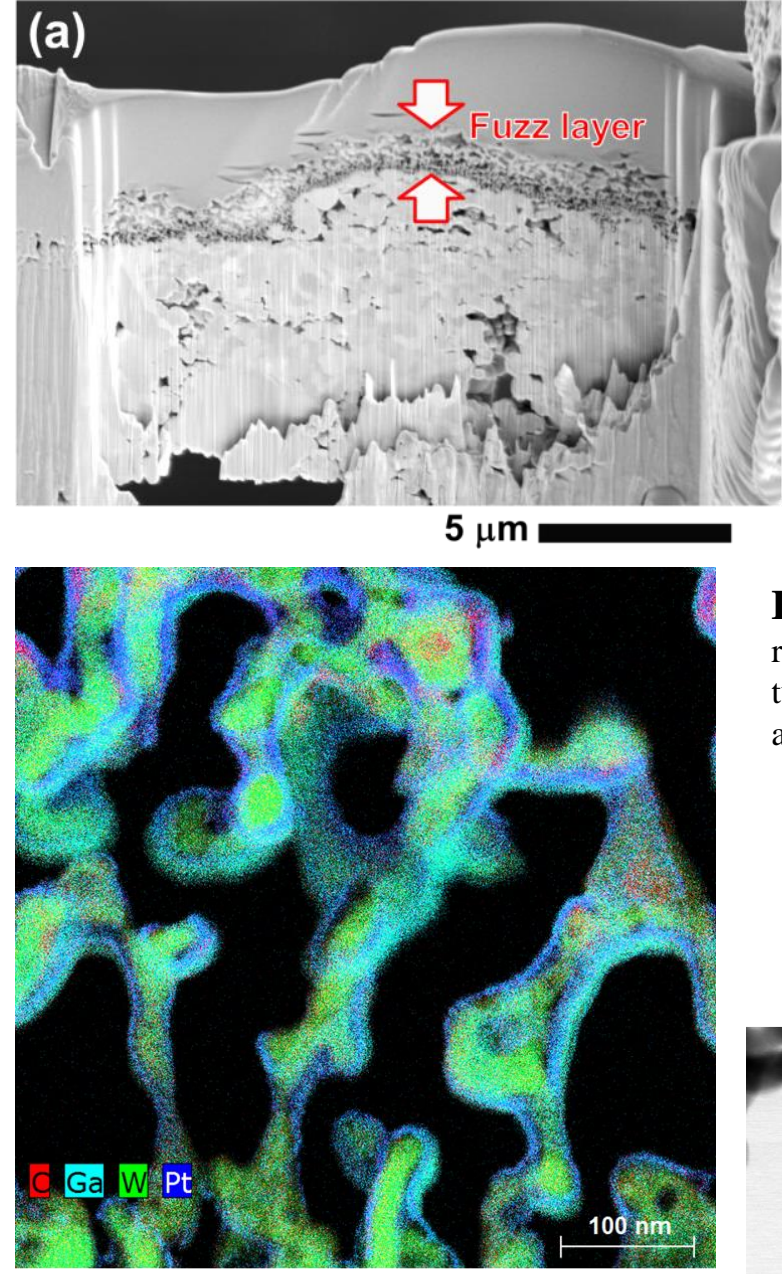

$100 \mathrm{~nm}$

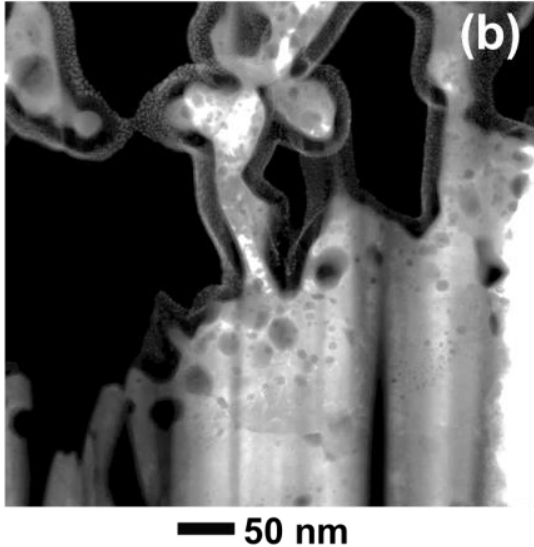

Figure 2: False-color Xray maps of FIB-prepared tungsten nanofuzz. FIB artifacts coat the tendrils.
Figure 1: (a) SEM image of FIB liftout from a tungsten nanofuzz sample. (b) STEM-HAADF image of the nanofuzz / substrate interface. Bubbles are visible deep in the substrate.

Figure 3: BF-STEM image of suspended nanofuzz. Circle denotes region of CBED pattern.
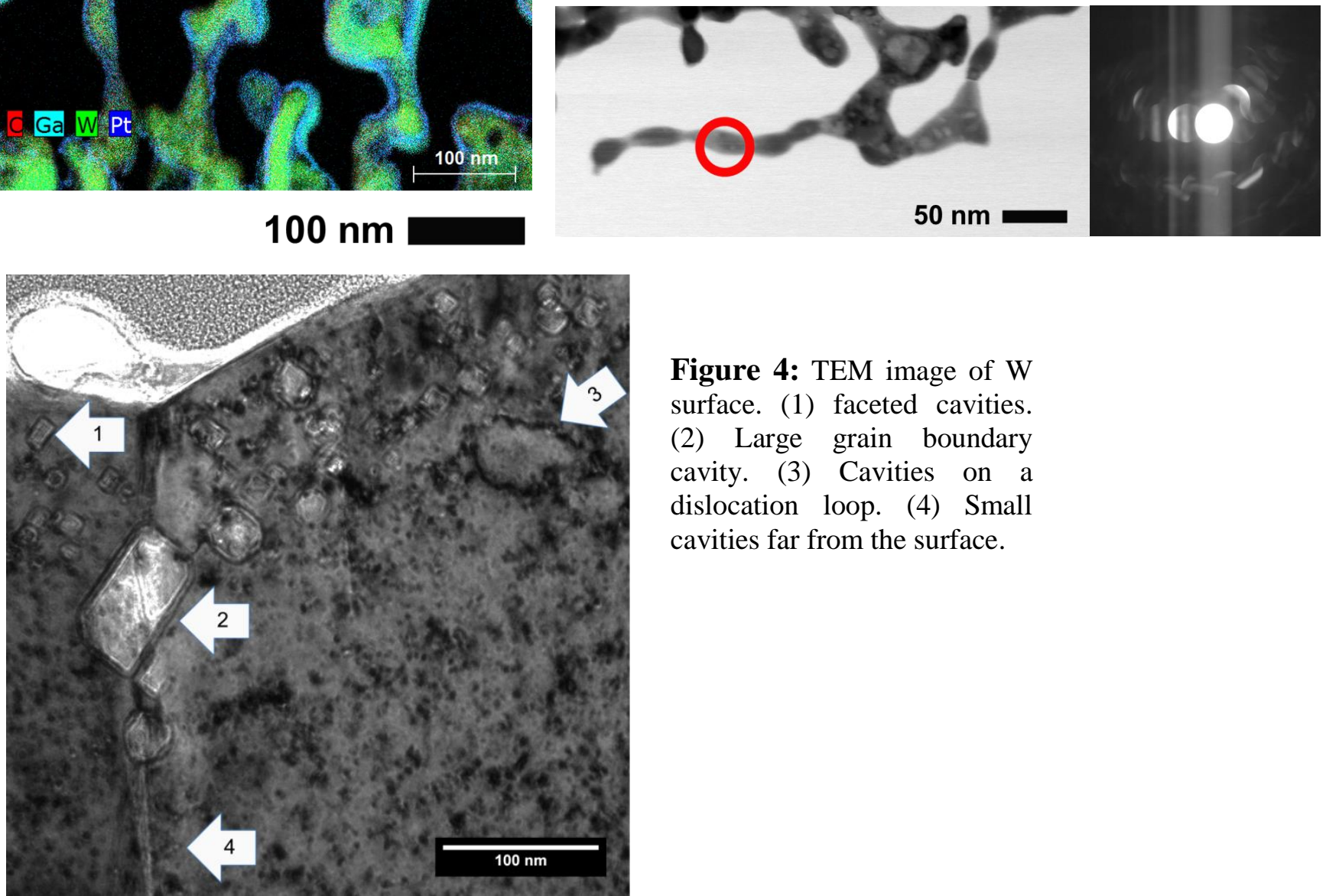

Figure 4: TEM image of $\mathrm{W}$ surface. (1) faceted cavities. (2) Large grain boundary cavity. (3) Cavities on a dislocation loop. (4) Small cavities far from the surface. 